\title{
Interpretation of information processing and mode of perception through the consciousness model involving though-carrying particle (TCP), thought retaining particle (TRP) and thought force $\left(T_{F}\right)$ in vitro and thought force $\left(\mathrm{T}_{\mathrm{F}}\right)$ in vivo
}

\section{Dhananjay Pal}

Pharmacy College, Bengal School of Technology, Sugandha-Delhi Road, Chuchura, Dist.-Hooghly, West Bengal, INDIA, PIN-712 102

Email address:

dhananjay.pal123@gmail.com,paldhananjay46@yahoo.com

\section{To cite this article:}

Dhananjay Pal. Interpretation of Information Processing and Mode of Perception Through the Consciousness Model Involving ThoughCarrying Particle (TCP), Thought Retaining Particle (TRP) and Thought Force (TF) in vitro and Thought Force (TF) in vivo. American Journal of Modern Physics. Vol. 2, No. 6, 2013, pp. 341-349. doi: 10.11648/j.ajmp.20130206.21

\begin{abstract}
A single field emerged at the origin of the universe, already containing within itself the blueprint of the physical universe. The primordial single field triggered the onset of the universe. Most physicists believe that a single super-force dominated the first instants of creation. Scientists have arrived at a simple but decisive conclusion that consciousness is very much a part of the universe, like other objects. Our consciousness model involving thought-carrying particle (TCP), thought retaining particle (TRP) and thought force (TF) signifies the existence of universal consciousness that exists along with the universe. This universal consciousness is a functional state of the universal mind. This universal mind (UM) is evolved at the Big Bang from void. The UM is constituted by these TCP and TRP in the inherent presence of thought force (TF). Thought force (TF) is an expression of universal consciousness. The Thought force (TF) being the primordial quantum field functions as the original super-force. TF being the original super-force functions as the origin of all the fundamental fields. TCP is the carrier of thought force (TF) that, in turn, appears to be the origin of all the fields. The quantized energy $\left(\varepsilon_{T}\right)$ of TCP is responsible to cause the universal consciousness as well as the cosmic microwave background radiation temperature. The individual consciousness owes its origin to the universal consciousness created by the same $\varepsilon_{T}$. The same $\varepsilon_{T}$ is the energy responsible for generating thought force (TF). TF being an expression of the universal consciousness is applicable to any inanimate object as well as to any biological system (having thinking ability). The TF exerts its functions both in vitro and in vivo. These TCP, TRP and the thought force (TF) in vitro and thought force (TF) in vivo play significant roles in information processing and mode of perception. The information is coming from the UM through TCP and TRP in biological systems.
\end{abstract}

Keywords: Void, Universal Mind (UM), Thought Force $\left(\mathrm{T}_{\mathrm{F}}\right)$, Though-Carrying Particle (TCP), Thought Retaining Particle (TRP), Quantized energy $\left(\mathcal{E}_{T}\right)$ of TCP

\section{Introduction}

In Eastern philosophical traditions, consciousness is intrinsic to the universe, whereas in most Western views, consciousness is extrinsic, emerging from complex computation. How can these views be reconciled?

It is most relevant and important to indicate the names of various eminent physicists like Erwin Schrödinger, Eugene Wigner, Brian Josephson, John Wheeler, Roger Penrose, Henry P Stapp, Freeman J. Dyson, Paul Davies, David
Bohm, Basil Hiley, Fritjof Capra, Fred Alan Wolf and Amit Goswami who have addressed the inclusion of consciousness in their work. Consciousness is to be taken into account.

Consciousness model of Pal et al [1-3] involving TCP, TRP and thought force $\left(\mathrm{T}_{\mathrm{F}}\right)$ signifies the existence of universal consciousness that exists along with the universe.

Bhaumik [4] mentioned, "It would be reasonable to presume that the universe originated in a unity of all fields at or near Planck's dimension. John Wheeler strongly believes that "in defining any useful concept of reality" we 
have to take into account "the indispensable place of the participating observer---evidenced in quantum mechanics," the foregoing cannot be really true unless consciousness is as essential as aspect of nature as are the fields that give rise to force and matter and the primary field that gave rise to them".

Physicists determined that underlying quantum fields give birth to elementary particles. Bhaumik [4] mentioned that Frank Wilczek pointed out, "In quantum field theory, the primary elements of reality are not individual particles, but underlying fields. Thus, for example, all electrons are but excitations of an underlying field, naturally called electric field". The same holds true for all the fundamental particles of which matter is made.

TCP cannot exist without TRP and vice versa. Many physicists believe that unifying all the forces, including gravity, into a single theory would require a phenomenon called super-symmetry. With super-symmetry, every fermion would have a boson twin, and vice-versa. TCP that behaves like boson should accompany its supersymmetrical partner TRP that functions like fermion in the generalized simpler way. Thus TCP like boson cannot have anti-particle. But TRP that functions like fermion should have its anti-particle and here it is shown as Anti-TRP. It is to be noted that these TCP and TRP function like wavicle: wave-particle duality.

1.1. In contrast to the usual linear sequence of matter, body, life, brain, mind, consciousness, here the proposed cyclic sequence is first universal consciousness (a functional state of the universal mind), and then matter, body, life, brain, and regeneration of mind and consciousness. The evolution of life with mind and consciousness is possible purely due to the inherent existence of universal consciousness which exists along with the universe. The human nervous system is evolved to provide an appropriate material structure to individualize the universal consciousness, a characteristic of reality, pervading all manifestations.

Consciousness model of Pal et al [1-3] involving TCP, TRP and thought force $\left(\mathrm{T}_{\mathrm{F}}\right)$ signifies the existence of universal consciousness that exists along with the universe. Pal et al [3] showed that this universal consciousness is a functional state of Universal Mind (UM). Pal et al [3] explained that the UM is evolved at the Big Bang from the eternal Void. This Void, in turn, is the source of infinite energy. And this $\mathrm{UM}$ is a finer matter. The individual mind being a constituent of the UM is also a finer matter. The constituents of the UM and individual mind are the same. The ultimate constituents of matter and mind are the same as both mind and matter are aspects of one fundamental reality, which is called UM. The brain is the mediating link or interface between the individual mind and body.

Pal et al [3] explained that the constituents of the UM are the ultimate constituents of matter itself as everything in this universe is a manifestation of this UM. Pal et al [3] expressed that the UM is constituted by these TCP and TRP in the inherent presence of thought force $\left(\mathrm{T}_{\mathrm{F}}\right)$. Pal et al $[1,3]$ further explained that the ultimate constituents of matter and mind are these TCP and TRP in the inherent presence of thought force $\left(\mathrm{T}_{\mathrm{F}}\right)$ in vitro and thought force $\left(\mathrm{T}_{\mathrm{F}}\right)$ in vivo. Pal [5] explained the existence of the thought force $\left(T_{F}\right)$ that, in turn, is the primordial quantum field.

1.2. Pal et al [3] developed three different equations expressing the quantized energy ( $\mathcal{E}_{T}$ ) of TCP. The value of $\mathcal{E}_{T}$ in one of the three equations is shown below:

$$
\varepsilon_{T}=4.384 \times 10^{-16} \mathrm{erg} \equiv 2.73 \times 10^{-4} \mathrm{eV} \equiv 2.73^{0} \mathrm{~K} \cong C M B R \text { temperature } \equiv 2.725^{\circ} \mathrm{K}
$$

It is to be noted that $1 \mathrm{erg} \cong 0.6241807 \times 10^{12} \mathrm{eV}$ and $10^{-4} \mathrm{eV \cong} 1^{\mathrm{O}} \mathrm{K}$ (where $\mathrm{K}=$ Kelvin) as indicated by Weisskopf [6].

1.3. Pal et al [3] expressed that the CMBR temperature is due to $\varepsilon_{T}$, the quantized energy of TCP where $\varepsilon_{T}=4.384 \times 10^{-16} \mathrm{erg} \equiv 2.73^{\circ} \mathrm{K}$ that is very close to $2.725^{\circ} \mathrm{K}$ (CMBR temperature). As per Pal et al [3], it is interesting to note that all of the three different equations ultimately give rise to the same result that is equivalent to the CMBR temperature. A sort of relationship is thus observed between the $\varepsilon_{T}$ and the CMBR temperature. This coincidence is thus signifying a probable role of TCP on the maintenance of CMBR temperature. Further, this coincidence is also signifying the existence of these TCP in the presence of TRP. TCP cannot exist without TRP and vice versa. The presence of TCP, TRP and Thought force $\left(T_{F}\right)$ in the universe is thus indicated and expressed mathematically. At present we are unable to explain when and how these TCP and TRP decoupled from the primordial cosmic soup.

Pal et al $[1,3]$ showed that the quantized energy $\left(\varepsilon_{T}\right)$ of
TCP is responsible to cause the universal consciousness as well as the cosmic microwave background radiation temperature. The individual consciousness owes its origin to the universal consciousness created by the same $\varepsilon_{T}$. Ultimately this $\varepsilon_{T}$ represents universal consciousness. The existence of CMBR temperature indicates the existence of the TCP in the presence of TRP. The existence of TCP ensures the existence of the thought force $\left(\mathrm{T}_{\mathrm{F}}\right)$. Further, this $\mathrm{T}_{\mathrm{F}}$ being an expression of the quantized energy ( $\varepsilon_{T}$ ) of TCP exerts its functions both in vitro and in vivo.

\section{Thought Force}

Pal [5] explained the existence of Thought force $\left(\mathrm{T}_{\mathrm{F}}\right)$. This Thought force $\left(\mathrm{T}_{\mathrm{F}}\right)$, an expression of the universal consciousness, is the primordial quantum field that, in turn, functions as the primary unified field. This $T_{F}$ being an expression of the universal consciousness is applicable to any inanimate object as well as to any biological system (having thinking ability). Thus the 
$\mathrm{T}_{\mathrm{F}}$ being an expression of the universal consciousness exerts its functions both in vitro and in vivo.

Physicists determined that underlying quantum fields give birth to elementary particles. Pal [5] expressed that the thought force $\left(T_{F}\right)$ is the primordial quantum field. Thought force $\left(\mathrm{T}_{\mathrm{F}}\right)$ being the primordial quantum field functions as the primary unified field. Thought force $\left(\mathrm{T}_{\mathrm{F}}\right)$ being the primordial quantum field gives birth to TRP that appears to be the origin of all the matter particles. TCP is the carrier of thought force $\left(\mathrm{T}_{\mathrm{F}}\right)$ that, in turn, appears to be the origin of all the fields. TCP thus appears to be the origin of all the field particles.

In a purpose to involve both the non-living and living systems of the world, Pal [5] has shown the existences of these TCP, TRP and thought force $\left(\mathrm{T}_{\mathrm{F}}\right)$ in vitro and thought force $\left(\mathrm{T}_{\mathrm{F}}\right)$ in vivo.

Pal (5) expressed that the non-living system of the world is governed by the thought force $\left(\mathrm{T}_{\mathrm{F}}\right)$ in vitro and this Thought force $\left(\mathrm{T}_{\mathrm{F}}\right)$ in vitro gives rise to $\mathrm{T}_{\mathrm{F}}$ (micro), $\mathrm{SNF}$, EMF, WNF, GF and $\mathrm{T}_{\mathrm{F}}$ (macro)

where $\mathrm{T}_{\mathrm{F}}$ (micro) $=$ Thought force in microcosm, $\mathrm{SNF}=$ Strong nuclear force, $\mathrm{EMF}=$ Electromagnetic force, $\mathrm{WNF}=$ Weak nuclear force, $\mathrm{GF}=$ Gravitational force and $\mathrm{T}_{\mathrm{F}}$ (macro) $=$ Thought force in macrocosm. It is to be noted here that $\mathrm{T}_{\mathrm{F}}$ (micro) is a stronger force than the SNF and $\mathrm{T}_{\mathrm{F}}$ (macro) is a weaker force even than the GF.

Pal (5) also expressed that the living system of the world is governed by the thought force $\left(\mathrm{T}_{\mathrm{F}}\right)$ in vivo and this Thought force $\left(\mathrm{T}_{\mathrm{F}}\right)$ in vivo is a type of force that represents the biological 'thought' which is the action of mind. This 'thought' being a type of force controls the 'thought processes' involving the firing of neurons through the quantum mechanical activities of these TCP and TRP in the presence of consciousness. Consciousness in living organisms is a process which involves the quantum mechanical activities of these TCP and TRP, the ultimate constituents of any matter as well as any mind in the inherent presence of thought force $\left(\mathrm{T}_{\mathrm{F}}\right)$ in vitro and the thought force $\left(\mathrm{T}_{\mathrm{F}}\right)$ in vivo as indicated by Pal [5]. These TCP and TRP govern the activities of neurons (not the other way round). Neurons are simply the equipments used to generate consciousness and awareness. This consciousness, in turn, is the quantized energy $\left(\varepsilon_{T}\right)$ of TCP.

The thought force $\left(\mathrm{T}_{\mathrm{F}}\right)$ in vivo is demonstrated in numerous experiments in which thought has an effect on a physical process (often known as mind over matter). This biological 'thought' is a type of force that can cause movement. Controlling movement through thought alone is observed in several experiments conducted by many scientists as indicated by Pal (5). These experiments thus signify the existence of thought force $\left(\mathrm{T}_{\mathrm{F}}\right)$ in vivo.

Pal [5] explained the existence of $\mathrm{T}_{\mathrm{F}}$ (micro) (= Thought force in microcosm). This $\mathrm{T}_{\mathrm{F}}$ (micro) is the strongest interaction (a new class of 'extra strong' interaction). It is stronger than SNF (Strong Nuclear Force).

Pal [5] has also shown the existence of $\mathrm{T}_{\mathrm{F}}$ (macro) (= Thought force in macrocosm). It is the "weakest force" which is much weaker even than the gravity.

\section{What is Information? What is Information Processing?}

\subsection{What is Information}

Information is a sequence of symbols that can be interpreted as a message. Information can be recorded as signs, or transmitted as signals. Conceptually, information is the message being conveyed. This concept of information is closely related to notions of communication, control, data, form, instruction, knowledge, meaning, mental stimulus, pattern, perception, representation, and especially entropy.

\subsection{Information Processing}

Information processing is the science concerned with gathering, manipulating, storing, retrieving, and classifying recorded information. It is the processing of information in any manner detectable by an observer. As such, it is a process that describes everything that happens in the universe, from the falling of a rock (a change in position) to the printing of a text file from a digital computer system.

The fundamental question of information philosophy is cosmological and ultimately metaphysical. What is the process that creates information structures in the universe? Given the second law of thermodynamics, which says that any system will over time approach a thermodynamic equilibrium of maximum disorder or entropy, in which all information is lost, and given the best current model for the origin of the universe, which says everything began in a state of equilibrium some 13.75 billion years ago, how can it be that living beings are creating and communicating new information every day? Why are we not still in that state of equilibrium?

When information is stored in any structure, two physical processes must occur. The first is the mysterious collapse of a quantum-mechanical wave function, which happens in any measurement process. Such quantum events involve irreducible indeterminacy and chance, but less often noted is the fact that quantum physics is directly responsible for the extraordinary temporal stability of most information structures.

The second is a local decrease in the entropy (which appears to violate the second law) corresponding to the increase in information. Entropy that is greater than the information increase must be transferred away, ultimately to the cosmic background, to satisfy the second law.

In less than two decades of the mid-twentieth century, the word information was transformed from a synonym for knowledge into a mathematical, physical, and biological quantity that can be measured and studied scientifically.

In information science, noise is generally the enemy of information. But some noise is the friend of freedom, since it is the source of novelty, of creativity and invention, and of variation in the biological gene pool. Too much noise is simply entropic and destructive.

In the late 1940s, Shannon developed the modern mathematical theory of the communication of information. Norbert Wiener wrote in his 1948 book Cybernetics that "information is the negative of the quantity usually defined 
as entropy," and in 1949 Leon Brillouin coined the term "negentropy."

The Information Philosopher website stated: Finally, in the early 1950s, inheritable characteristics were shown by Francis Crick, James Watson, and George Gamow to be transmitted from generation to generation in a digital code.

Information is neither matter nor energy, but it needs matter for its embodiment and energy for its communication. Immaterial information is perhaps as close as a physical scientist can get to the idea of a soul or spirit that departs the body at death. When a living being dies, it is the maintenance of biological information that ceases. The matter remains.

The Information Philosopher website further stated: Biological systems are different from purely physical systems primarily because they create, store, and communicate information. Living things store information in a memory of the past that they use to shape their future. Fundamental physical objects like atoms have no history. And when human beings export some of their personal information to make it a part of human culture, then this information moves closer to becoming immortal.

As per the Information Philosopher, human beings differ from other animals in their extraordinary ability to communicate information and store it in external artifacts. In the last decade the amount of external information per person has grown to exceed an individual's purely biological information. The new cognitive science likens the brain to a computer, with some programs and data inherited and others developed as appropriate reactions to experience.

But the brain may be regarded less as an algorithmic computer than as a primitive experience recorder and reproducer. Information about an experience - the sights, sounds, smells, touch, and taste - is recorded along with the emotions - feelings of pleasure, pain, hopes, and fears - that accompany the experience. When confronted with similar experiences later, the brain can reproduce information about the original experience (an instant replay) to guide current actions.

Information is constant in a deterministic universe. There is "nothing new under the sun." The creation of new information is not possible without the random chance and uncertainty of quantum mechanics, plus the extraordinary temporal stability of quantum mechanical structures.

It is of the deepest philosophical significance that information is based on the mathematics of probability. If all outcomes were certain, there would be no "surprises" in the universe. Information would be conserved and a universal constant, as some mathematicians mistakenly believe. Information philosophy requires the ontological uncertainty and probabilistic outcomes of modern quantum physics to produce new information.

But at the same time, without the extraordinary stability of quantized information structures over cosmological time scales, life and the universe we know would not be possible. Quantum mechanics reveals the architecture of the universe to be discrete rather than continuous, to be digital rather than analog.
The role of TCP and TRP in the presence of the thought force $\left(\mathrm{T}_{\mathrm{F}}\right)$ in vitro and thought force $\left(\mathrm{T}_{\mathrm{F}}\right)$ in vivo and their quantized information structures over cosmological time scales can provide plausible guidelines to evolve the universe with life.

Moreover, the "correspondence principle" of quantum mechanics and the "law of large numbers" of statistics ensure that macroscopic objects can normally average out microscopic uncertainties and probabilities to provide the "adequate determinism" that show up in all our "Laws of Nature."

In physics, information philosophy provides new insights into the problem of measurement, the paradox of Schrödinger's Cat, and finally information provides a better understanding of the entanglement and non-locality phenomena that are the basis for modern quantum cryptography and quantum computing.

In traditional philosophy, the total amount of information in the conceptually closed universe is static, a physical constant of nature. The laws of nature allow no exceptions, they are perfectly causal. Chance and change - in a deep philosophical sense - are illusions.

Monod [7] finds that teleonomic purposeful behavior is one of the fundamental characteristics of life, along with what he calls autonomous morphogenesis (life is "selfconstructing") and reproductive invariance (life is "selfreplicating").

Monod [7] says that some biologists have been unhappy with his idea of teleonomy that living beings are endowed with a purpose or a project, but he says this is essential to the definition of living beings. His next criterion is autonomous morphogenesis.

Information philosophy agrees that with the emergence of life, information structures with purposes entered the universe.

\subsection{The Information Processing Approach in Psychology}

The information processing approach in psychology is closely allied to the Computational theory of mind in philosophy; it is also related, though not identical, to cognitivism in psychology and functionalism in philosophy. In philosophy, the computational theory of mind is the view that the human mind is an information processing system and that thinking is a form of computing.

Within the field of cognitive psychology, information processing is an approach to the goal of understanding human thinking. The essence of the approach is to see cognition as being in essence computational in nature, with mind being the software and the brain being the hardware.

At the very heart of cognitive psychology is the idea of information processing. Cognitive psychology sees the individual as a processor of information, in much the same way that a computer takes in information and follows a program to produce an output.

3.4. Cognitive psychology compares the human mind to a computer, suggesting that we too are information processors and that it is possible and desirable to study the internal mental / mediational processes that lie between the stimuli (in our environment) and the response we make. 
The Information Processing System:

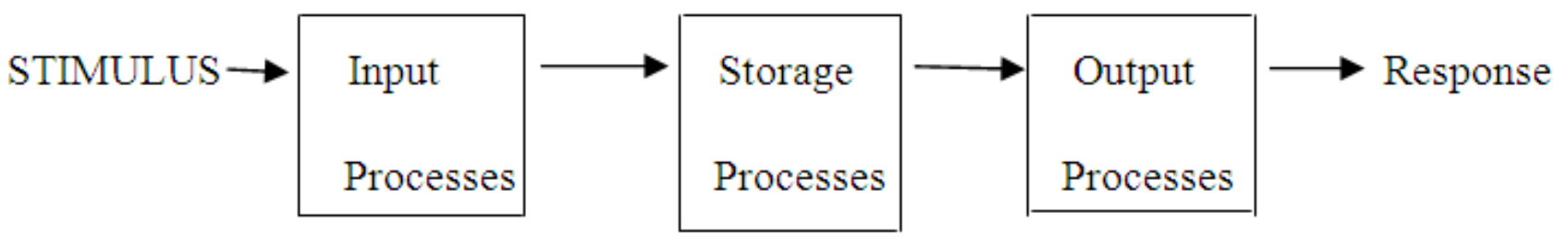

Information processing models consist of a series of stages, or boxes, which represent stages of processing. Arrows indicate the flow of information from one stage to the next.

* Input processes are concerned with the analysis of the stimuli.

* Storage processes cover everything that happens to stimuli internally in the brain and can include coding and manipulation of the stimuli.

* Output processes are responsible for preparing an appropriate response to a stimulus.

\subsection{Basic Assumptions of the Information Processing Approach to Cognitive Processes}

The information processing approach is based on a number of assumptions, including:

(1) information made available by the environment is processed by a series of processing systems (e.g. attention, perception, short-term memory);

(2) these processing systems transform or alter the information in systematic ways;

(3) the aim of research is to specify the processes and structures that underlie cognitive performance;

(4) information processing in humans resembles that in computers.

When we deal with information, we do so in steps. One way to think of this is to picture the process of acquiring, retaining, and using information as an activity called information processing. Information comes from the outside world into the sensory registers in the human brain. This input consists of things perceived by our senses. We are not consciously aware of most of the things we perceive; we become aware of them only if we consciously direct our attention to them. When we do focus our attention on them, they are placed in our working memory.

\section{Mode of Perception}

Regarding the perception, let us see Scheme-III for the explanation expressed by Sânkhya psychology as indicated by Vivekananda [8] In the case of vision, for instance, there are, first of all, the instruments of vision, the eyes. Behind the instruments (eyes), there is the organ of vision or 'Indria' (Sense organ), the optic nerve and its centers which are not the external instruments but without which the eyes will not see. The external sensations cannot be carried to the brain by the eyes alone if the sensory nerves (here optic nerve) and its centers are destroyed. Still more is needed for the perception. The mind (or Manas in Sanskrit) must come and attach itself to the organ to play its own role. Let us suppose that we are absorbed in reading a detective novel with close attention and if a bell rings at that time, we will not perhaps, hear the bell ring. The pulsation of that sound came to our ears, struck the tympanum; the impression was carried by the nerve into the brain. If the whole process was completed up ---catching, carrying and conducting the impulse to the brain, then why did we not hear? Something else was missing in the connection---the mind was not attached to the organ. When the mind detaches itself from the organ, the organ may bring any news to it, but the mind will not receive it. When mind attaches itself to the organ, then alone it is possible for the mind to receive the news. Yet, even that does not complete the whole circuit. The instrument may bring the sensation from the outside, the organ may carry it inside, the mind may attach itself to the organ, and yet the perception may not be complete. One more factor is necessary. There must be a reaction within. With this reaction comes knowledge. The sensation must be carried to the intellect (or BUDDHI), the determinative, reactive state of mind. The mind, after receiving the current of news from the brain, presents it to the intellect, which groups it in relation to the pre-received impressions and sends a current of reaction and perception becomes possible with that reaction. When the reaction comes from the intellect, along with it flashes the external world and egoism. Here then is the will. But everything is not complete. One more step is required. All the ideas in the mind must be gathered and projected on something that is stationary relatively to the body and mind. This internal 'something' stationary upon which our sensations, carried by the mind and intellect, are placed and grouped and formed into a unity, is what is called the Soul ( $\cong$ VOID), the master ruler of the body (see Scheme-III). The impulse

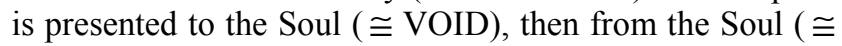
VOID) comes the order which goes down in the same sequence to the intellect, to the mind, to the brain, and the brain conveys it to the instruments through efferent nerve pathways and then the perception is truly complete (see Scheme-III). 


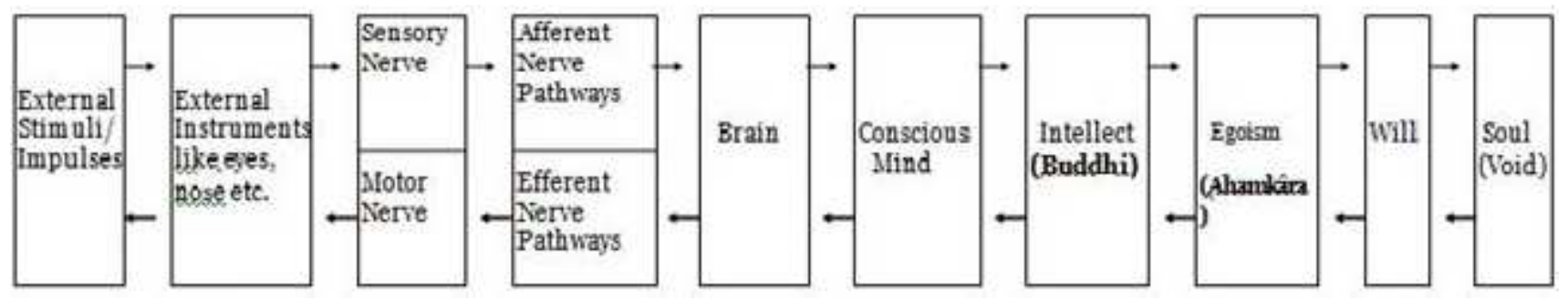

$(\rightarrow)$ indicates Sensory/Afferent Nerve conduction towards the brain.

$(\leftarrow)$ indicates Motor/Efferent Nerve conduction towards periphery.

Scheme-III. Schematic presentation showing the mode of perception:

According to the Sânkhya psychology as indicated by Vivekananda [8], the reactive state of mind called intellect (or BUDDHI) is the out-come or a certain manifestation of the cosmic mind (MAHAT) called the Universal Mind (UM).

\section{The Quantum Mechanical Activities of TCP and TRP in the Inherent Presence of the Thought Force $\left(T_{F}\right)$ in Vitro and Thought Force $\left(\mathrm{T}_{\mathrm{F}}\right)$ in Vivo Play Significant Roles in Information Processing}

We mentioned earlier that the role of TCP and TRP in the presence of the thought force (TF) in vitro and thought force (TF) in vivo and their quantized information structures over cosmological time scales can provide plausible guidelines to evolve the universe with life.

Overbye [9] stated: Perhaps the most mysterious and farreaching consequence of the exploding black hole is the idea that the Universe can be compared to a hologram, in which information for a three-dimensional image can be stored on a flat surface, like an image on a bank-card. "Entropy is a measure of how much information one can pack into an object," Bekenstein [10-12] explained. "The limit on entropy is a limit on information." This was a strange result. Normally one might think that there were as many choices -or degrees of freedom about the inner state of an object---as there were points inside that space. But according to Bekenstein bound, there were only as many choices as there were points on its outer surface. The 'points' in this case are regions with the dimensions of $10^{-33} \mathrm{~cm}$, the so-called Planck length that physicists believe are the 'grains' of space.

It is to be noted that the quantized energy ( $\left.\varepsilon_{T}\right)$ of TCP represents consciousness through which man recognizes the physical universe and react with it in accordance with the laws of physics. But serious problem arises when it is thought that the information (from external physical sources) trapped inside a biological system generates consciousness. In this case, it is the deepest problem man has ever encountered. Human failure to compare consciousness with anything visible or invisible has created a real crisis, to the extent that people react with disgust to the idea of defining consciousness with physical properties. It is extremely difficult to correlate the exact relationship between the brain as material body with the non-material consciousness. This deepest problem can be solved if we accept the existence of quantized energy $\left(\mathcal{E}_{T}\right)$ of TCP that represents universal consciousness. And the individual consciousness owes its origin to the universal consciousness created by the same $\varepsilon_{T}$. The living organisms born with their own inherent consciousness but with different degrees of consciousness depending on the complexity of the developed brain. This brain is the fine instrument through which mind (composed of TCP and TRP) exerts its functions. The functional state of mind is consciousness. It is to be further noted that with the emergence of life, information structures with purposes entered the universe and the information is coming from the UM through the TCP and TRP with which the UM is constituted.

The debatable point is how can something without a spatial location interact at a specific location in the brain by firing a particular neuron inside the brain? Physicists have been puzzled with the idea of matter being moved around by anything other than the real forces, in particular, forces that have origins in other material particles. The problem can be solved only if physical laws can be derived to establish a relationship between consciousness and other well-defined matter. The thought force (TF) and the developed consciousness model of $\mathrm{Pal}$ et al [3] may provide plausible guidelines to form such a relationship of consciousness with other well-defined matter. This consciousness model may also provide guidelines to form a possible gross bridge between mind and matter, present physics and cognitive science, psychology and natural sciences, classical physics and quantum physics.

Emotions, 'living force', mind, consciousness and soul are the characteristics of life. How all the characteristics of life are related to life on the basis of its evolution from the inanimate matter? Orders and arrangements of atoms and molecules are thought to be the main characteristics required to form molecules necessary to evolve 'life'. Physico-chemical properties of atoms and molecules are thought to be responsible for those orders and arrangements.

Ilya Prigogine $[13,14]$ is noted for his work on dissipative structures, complex systems, and irreversibility. He $[13,14]$ is famous for his "Self-Organization in NonEquilibrium Systems" and "From Being To Becoming". He won a Nobel Prize in 1977 for his work demonstrating the 
fact that certain chemical systems, called 'dissipative structures,' naturally increase rather than decrease their complexity without violating the Law of Entropy. They accomplish this by exchanging energy for order, so that overall entropy still increases even as order is produced.

But what is the driving force to develop and maintain all those orders and arrangements in order to evolve 'life'? Is there any purpose? Ordering effects must be the result of some kind of intelligence. It appears that the over-all-order and arrangements of atoms and molecules are governed by the quantum mechanical activities of these TCP and TRP as per the directive of the universal mind (UM). This UM, in turn, is evolved from the eternal Void indicated by Pal et al [3]. The universal consciousness that is a functional state of the UM functions as the evolutionary force to promote the bio-field.

As per Roger Penrose [15], consciousness is a part of the universe. Van De Bogart [16] explained, "Since consciousness is a part of the universe it then follows that all consciousness, and the universe, are of the same matrix of energy fields". Our consciousness model involving TCP, TRP and thought force (TF) signifies the existence of universal consciousness that exists along with the universe. This universal consciousness is a functional state of the universal mind (UM).

Biological system is a complex information processing system. Davies [17] wrote in his 'The Fifth Miracle', the "necessary powers to produce life" could be "already implicit in the laws of physics themselves. Imagine life emerging from a soup in the same dependable way that a crystal emerges from a saturated solution". Indeed, when we consider some of nature's universal constants, such as G (gravitational constant) and h (Planck's quantum constant), it is eerie to consider that life as we know it could not have evolved had any of these constants been different by even a miniscule amount. Does this tell us something about how closely life is connected to the laws of nature and that the evolution of life is predetermined? Davies [17] expressed that life's distinctiveness does not lie in its chemical properties but in its "informational properties". A living organism is a complex information processing system. Life was sparked not by a molecular maelstrom but, somehow, by the organization of information. The introduction of information into the molecule leads to increased order, and therefore less entropy. The molecule 'drinks' information from the surroundings to transform a chemical entity into a molecule of life. This process is remarkable because it goes against the second law of thermodynamics, which is a universal law.

Further, the values of the fundamental constants--- the parameters of particle physicists' "standard model" --- must adopt narrowly defined ranges for our life-supporting world to emerge. They arrange themselves precisely for the fostering of stars, and therefore life, within the universe. What caused this arrangement? Some would ask, "Who arranged them?" To some observers, the fine-tuning of the fundamental constants suggests the universe as an artifact: the universe had a creator. The universe bears the footprint of God which, in our view, is the eternal Void. And Stephen Hawking's phrase--the 'mind of God', in our view, is constituted and operated by these TCP and TRP in the presence of the thought force (TF) in vitro and thought force (TF) in vivo.

The key to understanding life lies in explaining how DNA was formed. What brought about the ordered structure of the DNA, why the code uses four types of bases (Adenine, Thymine, Guanine, Cytosine) and not three or five, what did the earliest DNA's look like etc. are among the questions to be answered. Wigner [18, 19] had explained that a physical system could not make a transition from a non-living to a living state without contravening the laws of quantum physics. The inter-atomic forces that form biological molecules like proteins and DNA/RNA must be quantum mechanical in nature. Thus quantum theory involving the quantum mechanical activities of these TCP and TRP may decidedly help in understanding the evolution of life and consciousness. Davies [17] concluded, "I am of the opinion that there remains a huge gulf in our understanding. It is not merely ignorance about certain technical details; it is a major conceptual lacuna-----we are missing something very fundamental about the whole business---a fully satisfactory theory of the origin of life demands some radically new ideas".

Our consciousness model involving TCP, TRP and thought force (TF) in vitro and thought force (TF) in vivo provides a simple and radically new idea to explain the evolution of universe along with life and consciousness where $\varepsilon_{T}$, the quantized energy of TCP represents universal consciousness.

Bhaumik [4] mentioned, "American psychologist William James stated, "There is only one primal 'stuff' of which everything is composed. David Bohm and Basil Hiley said that at some level of reality (in the pre-space / implicate order), mind and matter merge. Bhom maintained that implicit in the quantum potential was a "mind-like quality in matter," which he identified with the notion of active information. Even an electron, he argues, has a rudimentary "mental" aspect. If this is true, it's reasonable to ask whether our brains, as material outgrowths of this process, have evolved to operate in perfect resonance with this active information. "One could say," Bohm and Hiley allow, "that through the human being, the universe is making a mirror to observe itself."

The existence of TCP, TRP and thought force (TF) in vitro and thought force (TF) in vivo may provide plausible guidelines to lucidly explain the evolution of universe with laws of natural science like physics, chemistry and biology (with consciousness). 


\section{The Role of TCP, TRP and Thought Force $\left(T_{F}\right)$ in Information Processing: These TCP and TRP are Inter-Convertible in the Presence of Consciousness to Carry and Retain a Specific "Thought" and Also for its Communication from one Person to Another in the Presence of the Thought Force $\left(\mathrm{T}_{\mathrm{F}}\right)$ in Vitro and Thought Force $\left(\mathrm{T}_{\mathrm{F}}\right)$ in Vivo}

Pal et al [3] expressed that TCP and TRP are the ultimate constituents of any matter as well as any mind in the inherent presence of the thought force $\left(\mathrm{T}_{\mathrm{F}}\right)$ in vitro and thought force $\left(\mathrm{T}_{\mathrm{F}}\right)$ in vivo.

Pal et al [3] expressed that consciousness in living organisms is a process which involves the quantum mechanical activities of these TCP and TRP, the ultimate constituents of matter and mind in the inherent presence of thought force $\left(\mathrm{T}_{\mathrm{F}}\right)$ in vitro and thought force $\left(\mathrm{T}_{\mathrm{F}}\right)$ in vivo. And these TCP and TRP govern the activities of neurons (not the other way round). These neurons are simply the equipments used to generate consciousness and awareness. The consciousness itself is functioning as an inter-linking agent between the animate and inanimate.

Pal et al [1,3] explained that analysis of consciousness and light yields many interesting and compelling parallelisms that are useful in trying to develop a theory of consciousness. Both the consciousness (thought) and light (waves and photons) function as 'carriers' of information and action, and both appear to be self-referential. Consciousness may thus be defined as a mental light through which a blind person can recognize any object through the use of his / her mental light. Pal et al [3] explained that the quantized energy ( $\mathcal{E}_{T}$ ) of TCP is related with c (free-space velocity of light) through the expression: $\mathcal{E}_{T}=\mathrm{hc} / \lambda_{\mathrm{T}}$.

'Thought' is a kind of force being carried by the TCP. A TCP has the capacity to carry a specific 'thought'. This TCP is converted into a TRP in the presence of consciousness to retain and store a specific 'thought' (or 'idea') as both these TCP and TRP are interchangeable in the presence of consciousness.

Thus, in the presence of consciousness, these TCP and TRP are inter-convertible to carry and retain a specific "thought" and also for its communication from one person to another in the presence of the thought force $\left(\mathrm{T}_{\mathrm{F}}\right)$ in vitro and thought force $\left(\mathrm{T}_{\mathrm{F}}\right)$ in vivo.

\section{Discussion and Conclusion}

\subsection{Discussion}

Transformation of energy waves into consciousness require an intermediate material stage, probably in the model of energy to matter and then from matter to the onset of consciousness in biological organisms. The brain as a biological system converts the energy waves into chemical energy in the material form through the highly electrolytic neuro-chemicals. These chemicals get ionized in various degrees depending upon the energy input, coming out as information wave of specific length and frequency of the TCP, and facilitating the transport of information to different segments of the brain for interpretation. The quantized energy $\left(\mathcal{E}_{T}\right)$ of the TCP is $4.384 \times 10^{-16} \mathrm{erg}$. The electrical activities inside the brain during the processing of information can be detected through Positron Emission Tomography and functional Magnetic Resonance Imaging (fMRI) in a target to find out the presence and role of these TCP and TRP.

\subsection{Conclusion}

The evolution of life having mind and consciousness is possible purely due to the ultimate fate of the eternal

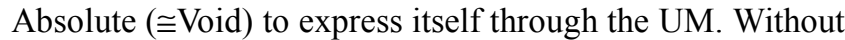
the inherent existence of the universal consciousness, the inanimate matter itself can not generate consciousness which is an inter-linking agent between the inanimate and animate. And further, the universal consciousness is a functional state of the UM which is constituted by these TCP and TRP which, in turn, are the ultimate constituents of matter and mind. This UM along with the universal consciousness is thus responsible for the manifestation of this Universe with all of its inhabitants. As this UM manifests its own inherent characteristics through the generations of each and all individual minds in a purpose to realize the "reality" of the universe itself, so the biogenesis may be thought to be predetermined. A living organism is thus a complex information processing system and the information is coming from the UM through TCP and TRP.

Hawking [20] mentioned: The Heisenberg's uncertainty principle established that the Void is filled with infinite pairs of virtual particles and antiparticles. These pairs would have an infinite amount of energy and therefore, by Einstein's equation: $E=m c^{2}$, they would have an infinite amount of mass.

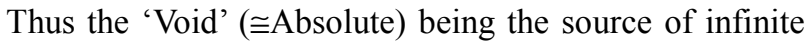
energy is the origin of anything and everything in this universe through the presence of universal consciousness. Therefore, the natural physical laws governed by the eternal 'Void' (Absolute) cause the evolution of life with consciousness through the generation of DNA and RNA by the quantum mechanical activities of these TCP and TRP which are conceived to be not only the constituents of the UM but also the ultimate constituents of matter and mind.

\section{References}

[1] D. Pal and A.U. De, Physics of consciousness and its model may provide guidelines to solve many scientific problems. Neuroquantology 1, 17-28 (2004) 
[2] D. Pal, and A.U. De, Consciousness model: Significance of thought-carrying particles and thought-retaining particles in quantum measurement as well as cognitive problem. NeuroQuantology 2, 115-116 (2005)

[3] D. Pal, and A.U. De, The cosmic microwave background radiation temperature signifying the existence of the thought-carrying particle, thought retaining particle and thought force. NeuroQuantology 10: Issue3; 428-442 (September 2012)

[4] M. Bhaumik, Code Name GOD. (Penguin Books India Pvt. Ltd., 11 Community Centre, Panchsheel Park, New Delhi 110 017, India) pp. 132-133; 161-162; 167; 171; 177; 183; 185-186; 89-190; 198 (2006)

[5] D. Pal, Existence of thought force and its characteristics. Communicated to American Journal of Modern Physics (AJMP) (2013)

[6] V. F. Weisskopf, The Origin of the Universe, The World of Physics, (Simon and Schuster, 1230 Avenue of Americas, New York 10020) 3, pp. 10 (1987)[7] JL. Monod, Chance and Necessity: An Essay on the Natural Philosophy of Modern Biology. (Alfred A. Knopf 1971). ISBN 0-39446615-2.

[7] JL. Monod, Chance and Necessity: An Essay on the Natural Philosophy of Modern Biology. (Alfred A. Knopf 1971). ISBN 0-394-46615-2.

[8] Swami Vivekananda, The Vedanta Philosophy. The real and the apparent Man. The complete works of Swami Vivekananda. (Advaita Ashrama 1989) 1: P-359-360; The Real and the Apparent Man, ibid.; 2: P-263.

[9] D. Overbye, Mysteries of the Universe. (Copyright 2002) The New York Times Company
[10] JD. Bekenstein, Entropy content and information flow in systems with limited energy. Phys Rev D (198 30:16691679

[11] JD. Bekenstein and M. Schiffer, Quantum Limitations on the Storage and Transmission of Information. Int $\mathrm{J}$ of Modern Physic (1990) 1:355-422

[12] JD. Bekenstein, Information in the Holographic Universe. (Scientific American 2003) Volume 289, Number 2: 61

[13] I. Prigogine and G. Nicolis, Self-Organization in NonEquilibrium Systems. (Wiley 1977) ISBN 0471024015.

[14] I. Prigogine, From Being To Becoming. (Freeman 1980). ISBN 0716711079.

[15] R. Penrose, Shadows of the Mind: A Search for the Missing Science of Consciousness. (Oxford University Press 1994)

[16] W. Van De Bogart, Earth portals: Exploring New Metaphors of Consciousness 1993 willard@earthportals.com

[17] P. Davies, The Fifth Miracle: The Search for the Origin and Meaning of Life. (Simon \& Schuster 1999)

[18] E. Wigner, Symmetries and Reflections: Scientific Essays. MIT Press. 1970.

[19] E. Wigner, "Remarks on the Mind-Body Problem", in The Scientist Speculates, I. J. Good, ed. pp. 284-302, Heinemann, London (1961); Basic Books, New York (1962). Reprinted in Wigner 1967/1983

[20] S. W. Hawking, A Brief History of Time from the Big Bang to Black Holes. (Bantam Books, 666 fifth Avenue, New York 10103) pp. 143-144 and 165 (1989) 